\title{
A self-oscillating h.f. power generator with a Class E resonant amplifier
}

\author{
M. MIKOLAJEWSKI* \\ Institute of Radioelectronics, Warsaw University of Technology, 15/19 Nowowiejska St., 00-665 Warsaw, Poland
}

\begin{abstract}
Self-oscillating h.f. power generators with a Class E amplifier are used as industrial high-efficiency h.f. power sources. In the paper a modification of the classic Class E generator by applying an additional capacitor in the matching and feedback circuit is presented. The proposed solution increases the degree of freedom in the generator design and can noticeably reduce its cost by eliminating the need for the use of high-value h.f. capacitors in the feedback loop divider. The self-oscillating Class E generator $(150 \mathrm{~W} / 6.78 \mathrm{MHz}) \mathrm{with}$ the proposed modification has been designed, built and its parameters were measured. Theoretical predictions were in a good agreement with experimental results. Moreover, the burst control method of the output power in the generator has also been presented.
\end{abstract}

Key words: Class E amplifier, self-oscillating power generator, output power control.

\section{Introduction}

In many industries h.f. power amplifiers and generators find applications in induction and dielectric heaters, plasma generators, electronic ballasts for fluorescent and HID lamps, energy harvesting circuits as well as resonant $\mathrm{dc} / \mathrm{dc}$ converters [1-10]. Among possible solutions amplifiers and generators utilizing high-efficiency resonant switching Class DE and Class E amplifiers are particularly attractive. Resonant shaping of current and/or voltage waveforms across transistor switches (ZCS/ZVS switching) in these amplifiers significantly reduces switching losses increasing their power efficiency up $95-98 \%$ in practical applications $[11,12]$. This decreases the cost of both used transistor switches and their cooling. Moreover, due to high efficiency the circuit miniaturization is also increased.

An essential problem in the application of Class DE and Class $\mathrm{E}$ amplifiers in the high-frequency range results from the switching of their transistors (often MOSFETs) which usually require driving of their gates with a rectangular, highamplitude waveform $v_{G S}(t)$ (a typical amplitude of the gate waveform $V_{G S m} \geq 10 \mathrm{~V}$ ). Such a method of transistor switch driving needs charging and discharging of the large MOSFET input capacitance (often of the order of nanofarads) by highamplitude current pulses and can cause power losses both in the driver circuit and the transistor gate resistance diminishing efficiency. These driving related power losses are proportional to the circuit operation frequency and the input capacitance of the used transistor switches. It becomes a major obstacle in designing switching power amplifiers operating at the frequencies above a few MHz and with output power beyond a few tens of watts. The problem is usually solved by the application of quasi-resonant or resonant gate drivers, which reduces required driving power and thus increases the operation frequency of the amplifiers to many $\mathrm{MHz}$ [9]. A disadvantage of this approach is the complexity of driver circuits caused by the use of additional diodes, transistors and reactive components. Moreover, in quasi-resonant and resonant drivers to compensate for the slow rise and fall of the gate voltage waveform its amplitude is usually higher than in the non-resonant gate drivers with rectangular waveforms. This increases conduction losses in resonant drivers and can breakdown the transistor if the amplitude of its gate voltage waveform exceeds the transistor maximum rating.

A simpler method of resonant driving of transistor switches is used in self-oscillating power generators with a Class DE or Class E amplifier. In these generators the h.f. sinusoidal gate voltage is supplied to the transistor gate from the amplifier resonant output circuit by means of a reactive positive feedback network $[11,13,14]$. Such generators can operate with a high frequency and high efficiency limited only by the parameters of the used transistor switches and the required output power. A disadvantage of the self-oscillating power generators is the sensitivity of the gate voltage waveform to the changes of both the amplifier dc supply voltage and the impedance of the amplifier resonant circuit. These changes have to be controlled to avoid the operation of the amplifier transistors in non-ZVS conditions, which increase switching losses in transistors and lower the circuit efficiency. This phenomenon also makes the control of output power in self-oscillating generators a difficult problem.

Output power control in a h.f. self-oscillating power generator is usually obtained by changing its operating frequency, by regulating its dc supply voltage or by the burst control method. Electronic frequency modulation in a self-oscillating power generator by changing values of capacitors or inductors in a resonant circuit of the generator is difficult to achieve in practice because of high voltages and currents occurring in the components [15]. Therefore, the most often used method of

\footnotetext{
*e-mail: M.Mikolajewski@ire.pw.edu.pl
} 
M. Mikołajewski

output power control is by regulation of the dc supply voltage by means of an additional power supply. This, however, rises the cost and complexity of the generator as well as reduces its total efficiency. In the burst control method the oscillations in the generator are started and stopped periodically at the frequency many times lower than the oscillation frequency. By changing the ratio of the generator turn-on time to its turn-off time the output power can be controlled in a wide range with high efficiency $[16,17]$.

In the classic Class E self-oscillating power generator [11, $13,14]$ its main advantage is high efficiency and simplicity of the resonant and feedback loop circuits. However, in some applications a more complex resonant network in the generator is desirable to optimize its operation as it has been shown in a self-oscillating electronic ballast with a Class E amplifier [3].

In the paper a self-oscillating Class $\mathrm{E}$ generator with a modified matching and feedback network is analyzed. The circuit eliminates the need to use expensive high-capacitance h.f. capacitors in the feedback signal divider of the generator. The proposed modification by reducing the values of capacitors in the gate circuit of the transistor switch simplifies also the application of the burst control of output power in the generator. A design method and results of the circuit experimental verification for a $150 \mathrm{~W} / 6.78 \mathrm{MHz}$ self-oscillating generator with a Class E amplifier are also given.

\section{A self-oscillating Class $\mathbf{E}$ generator}

A self-oscillating Class E generator is shown in Fig. 1a. The circuit consists of a Class $\mathrm{E}$ amplifier with the bi-directional transistor switch $T$, supply choke $L_{C H}$ and output resonant circuit $C_{R}-L_{S R}-C_{S R}-R_{L}$. The sinusoidal output voltage $v_{O}$ at the operating frequency $f$ is supplied to the capacitor voltage divider $C_{1}-C_{2}$. The divider along with inductance $L_{f}$ comprises a positive feedback loop network driving the gate of the switch $T$ with $v_{G S}(t)$ voltage waveform. The amplitude $V_{G S m}$ of the gate voltage waveform is high enough to switch transistor $T$ and the drain-to-gate phase shift for the fundamental component in the generator network satisfies phase conditions for oscillations. Dc voltage source $V_{G}$ via resistance $R_{G}$ sets the dc gate voltage $V_{G S 0}$ equal to the switch threshold voltage $V_{G S t h}=V_{G S 0}=V_{G}$, which initiates oscillations in the circuit with transistor normalized on-time $D=t_{O N} f=0.5$. Capacitor $C_{O}$ is the main reactive component which matches usually standard load resistance $R_{L}$ to $R_{o p t}$ - resistance required in the Class $\mathrm{E}$ amplifier to obtain assumed output power at the given value of dc supply voltage $V_{D D}$. The use of capacitor $C_{O}$ ensures that the values of capacitors in the divider $C_{1}-C_{2}$ can be small. Bi-directional Zener diode $D_{Z}$ protects the switch gate from breakdown by a high value of $V_{G S m}$.

In a classic self-oscillating Class $\mathrm{E}$ generator (without capacitor $C_{O}$ ) the feedback signal divider $C_{1}-C_{2}$ is also a matching circuit for load resistance, which causes that even for low output power the value of capacitor $C_{2}$ can exceed hundreds of nanofarads [11]. Because $C_{1}$ and $C_{2}$ usually conduct high-amplitude currents it is necessary to limit conduc- tion losses in them by applying h.f. low-loss expensive mica or porcelain capacitors as $C_{1}, C_{2}$. Typical values of available mica or porcelain capacitors do not exceed 10 nanofards. Therefore, high values of capacitance are obtained by paralleling many capacitors. This can significantly increase the cost of a classic Class E generator.

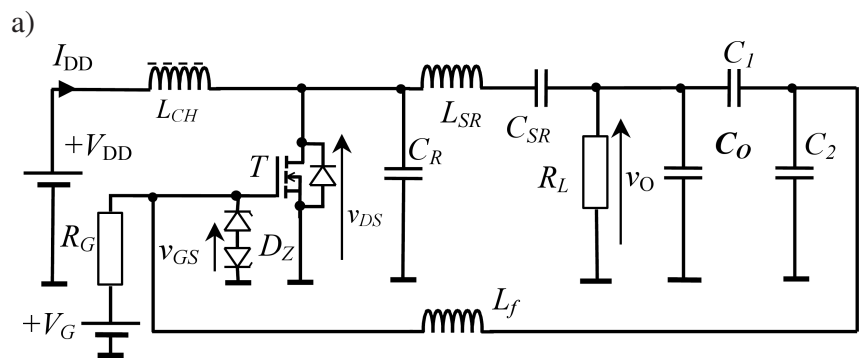

b)

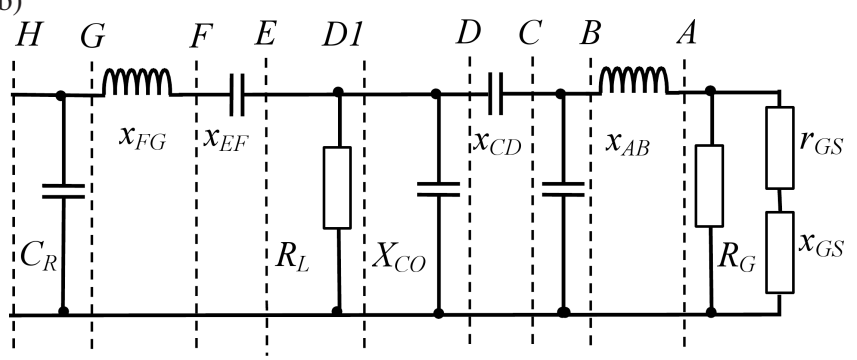

Fig. 1. The proposed self-oscillating Class E generator (a), equivalent circuit of the output and feedback network: $C_{O}$ - additional matching capacitor, $r_{G S}, x_{G S}$-series resistance and reactance of the gate circuit, respectively (with taking into account diode $D_{Z}$ capacitance)

\section{Experimental circuit - design and measurements}

To test the properties of the proposed modification in the self-oscillating Class E generator, the circuit of Fig. 1a was designed by means of the design procedure for the classic Class E generator given in [11]. In this procedure the resonant circuit and the feedback network are divided into sections (Fig. 1b). For the fundamental component for each section its input impedance (to the right of the section line) and its introduced phase shift are found. To achieve the operation of the generator at the desired frequency with high efficiency and demanded output power level it is necessary to satisfy both Barkhausen criteria $[18,19]$ and requirements for the nominal or off-nominal operation of the Class E amplifier [11, 12, 14].

In the proposed circuit an additional section $D D 1$ is introduced as shown in the equivalent circuit in Fig. $1 \mathrm{~b}$ to take into account capacitor $C_{O}$. A parameter $k$ is defined as a ratio of parallel reactances

$$
\begin{gathered}
k=\frac{X_{E}}{X_{C_{O}}}, \\
1-k=\frac{X_{E}}{X_{D}},
\end{gathered}
$$

where $X_{E}, X_{D}$ each is an input parallel reactance to the right of $E$ and $D$, respectively, $X_{C O}=1 /\left(2 \pi f C_{O}\right)$ is the 


\section{A self-oscillating h.f. power generator with a Class E resonant amplifier}

reactance of capacitor $C_{O}$ at the operation frequency $f$. The value of $k$ belongs to the range $0 \leq k<1$. Thus for $k=0$ is $X_{E}=X_{D}$ and $C_{O}=0$, and for $k \rightarrow 1$ is $X_{C O} \rightarrow X_{E}$, $X_{D} \rightarrow \infty$ and $C_{1} \rightarrow 0, C_{2} \rightarrow 0$. Therefore using (1), (2) and the design procedure [11] for the assumed $k$ the desired values of capacitors $C_{1}, C_{2}, C_{O}$ can be found which are small enough to ensure that the applied capacitors are both small in size and inexpensive.

To illustrate the application of the design method described in [11] to the proposed circuit, a design procedure for the experimental circuit is given. The presented procedure shows how the circuit modifications have been included into the existing design method.

The proposed generator was designed for the following assumptions:

- output power $P_{o}=150 \mathrm{~W}$, operating frequency $f=$ $6.78 \mathrm{MHz}$

- power efficiency $\eta=0.91$,

- the supply voltage $V_{D D}=48 \mathrm{~V}$, supply choke $L_{C H}=$ $10 \mu \mathrm{H}$,

- amplitude of the gate voltage waveform $V_{G S m}=13 \mathrm{~V}$,

- loaded quality factor of the Class E amplifier series branch $Q_{S R}=5$,

- load resistance $R_{L}=50 \Omega$,

- series loss resistance in inductances $L_{S R}$ and $L_{f}$ estimated as $r_{S R}=0.22 \Omega$ and $r_{f}=0.085 \Omega$, respectively,

- parameter $k=0.95$,

- as the switch $T$ transistor DE275-501N16A (IXYS) with Zener diode $D_{Z}$ (two back to back connected BZX85/C18 diodes) were used. Measured transistor $T$ threshold voltage was $V_{G S t h}=4.8 \mathrm{~V}=V_{G}$. The series gate resistance and reactance measured with diode $D_{Z}$ connected to the transistor $T$ gate were $r_{G S}=0.3 \Omega, x_{G S}=-8.2 \Omega$. From the manufacturer data the transistor $T$ on-resistance was $r_{D S}=0.4 \Omega$. The transistor output capacitance was assumed to be a part of capacitor $C_{R}$,

- resistance $R_{G}=2 \mathrm{k} \Omega$ was chosen to be high enough not to cause too much power loss in the gate circuit.

The power $P_{S}$ supplying the generator comprises output power $P_{O}$ and power losses $P_{l}$ resulting from losses in the circuit components. Because the value of $P_{l}$ is unknown at the beginning of calculations therefore $P_{S}$ is estimated by using the assumed power efficiency $\eta$.

$$
P_{S}=P_{O}+P_{l}=\frac{P_{O}}{\eta}=\frac{150}{0.91}=164.835 \mathrm{~W} .
$$

The values of series load resistance $R_{\text {opt }}$, parallel reactance $X_{C R}$, and capacitance $C_{R}$ for the nominal operation of the Class E amplifier with the supply power $P_{S}$ is found from formulas given in [12] as follows:

$$
\begin{gathered}
R_{o p t}=\frac{8}{\pi^{2}+4} \frac{V_{D D}^{2}}{P_{S}}=\frac{8}{\pi^{2}+4} \times \frac{48^{2}}{164.835}=8.0623 \Omega, \\
X_{C R}=-\frac{\pi\left(\pi^{2}+4\right)}{8} R_{o p t} \\
=-\frac{\pi\left(\pi^{2}+4\right)}{8} \times 8.0623=-43.912 \Omega,
\end{gathered}
$$

$$
\begin{gathered}
C_{R}=\frac{1}{2 \pi f\left|X_{C R}\right|} \\
=\frac{1}{2 \pi \times 6.78 \times 10^{6} \times 43.912}=534.57 \mathrm{pF} .
\end{gathered}
$$

Resistance $R_{\text {opt }}$ is equal to the input series equivalent resistance $r_{G}$ to the right of the section $G$. Reactance $X_{C R}$ is the parallel reactance of section $G H$

$$
\begin{gathered}
r_{G}=R_{o p t}, \\
X_{G H}=X_{C R} .
\end{gathered}
$$

The reactance factor $q_{G}$ for the $\mathrm{G}$ section of the Class $\mathrm{E}$ amplifier in the nominal condition is found as [12]

$$
q_{G}=\frac{x_{G}}{r_{G}}=\frac{\pi \times\left(\pi^{2}-4\right)}{16}=1.1525 .
$$

Thus, the input series reactance $x_{G}$ is

$$
x_{G}=q_{G} r_{G}=1.1525 \times 8.0623=9.2918 \Omega .
$$

For the assumed value of $Q_{S R}$ and resistance $r_{G}$ given by (7) the inductance $L_{S R}$ is

$$
L_{S R}=\frac{Q_{S R} r_{G}}{\omega}=\frac{5 \times 8.0623}{2 \pi \times 6.78 \times 10^{6}}=946.3 \mathrm{nH} .
$$

The reactance of the section $F G$ is equal to the reactance of $L_{S R}$

$$
x_{F G}=\omega L_{S R}=Q_{S R} r_{G}=5 \times 8.0623=40.312 \Omega .
$$

The series resistance $r_{E}$ to the right of the section $E$ is found by subtracting from $r_{G}$ the loss resistance of inductance $L_{S R}$

$$
r_{E}=r_{G}-r_{S R}=8.0623-0.22=7.8423 \Omega .
$$

The input series reactance $x_{F}$ to the right of the section $F$ equals to

$$
x_{F}=x_{G}-x_{F G}=9.2918-40.312=-31.020 \Omega .
$$

Load resistance $R_{L}$ equal to the parallel resistance $R_{D 1 E}$ of the section $D 1 E$ is shunted by the parallel resistance $R_{D 1}$ to the right of section $D 1$. The resistance $R_{D 1}$ results from the losses in inductance $L_{f}$ and the transistor $T$ gate circuit (assuming that losses in capacitors $C_{1}, C_{2}$ and Zener diode $D_{Z}$ are negligible).

The resultant input parallel resistance $R_{E}$ to the right of the section $E$ is a parallel connection of $R_{L}$ and $R_{D 1}$

$$
R_{E}=\frac{R_{L} R_{D 1}}{R_{L}+R_{D 1}}=\frac{R_{D 1 E} R_{D 1}}{R_{D 1 E}+R_{D 1}} .
$$

The power $P_{E}$ lost in $R_{E}$ comprises output power $P_{O}$ lost in $R_{L}$ and power $P_{D 1}$ lost in $R_{D 1}$.

$$
\begin{gathered}
P_{E}=P_{O}+P_{D 1}, \\
P_{O}=\frac{V_{O}^{2}}{R_{L}}=\frac{V_{O}^{2}}{R_{D 1 E}}, \\
P_{D 1}=\frac{V_{O}^{2}}{R_{D 1}}=P_{L f}+P_{A},
\end{gathered}
$$

where $P_{L f}$ - power loss in series parasitic resistance $r_{f}$ of inductance $L_{f}, P_{A}$ - power loss in section $A$ caused by resistor $R_{G}$ and the loss in the transistor gate. 
M. Mikołajewski

To estimate power losses $P_{L f}$ and $P_{A}$ it is necessary first to calculate the amplitude $I_{A m}$ of the current flowing into section $A$ and the input series resistance $r_{A}$ resulting from losses in the gate circuit.

For the transistor $T$ gate circuit the reactance factor $q_{G S}$ equals

$$
q_{G S}=\frac{R_{G S}}{X_{G S}}=\frac{x_{G S}}{r_{G S}}=\frac{-8.2}{0.3}=-27.333 \Omega .
$$

The input parallel gate resistance $R_{G S}$ and reactance $X_{G S}$ are found by transforming the input series gate resistance $r_{G S}$

$$
\begin{gathered}
R_{G S}=r_{G S}\left(1+q_{G S}^{2}\right) \\
=0.3 \times\left(1+(-27.333)^{2}\right)=224.43 \Omega, \\
X_{G S}=\frac{R_{G S}}{q_{G S}}=\frac{224.43}{-27.333}=-8.211 \Omega .
\end{gathered}
$$

The input parallel resistance $R_{A}$, input parallel reactance $X_{A}$ and the reactance factor $q_{A}$ of section $A$ result from the parallel connection of resistance $R_{G}$ as well as the input parallel resistance $R_{G S}$ and reactance $X_{G S}$ of the transistor gate.

$$
\begin{gathered}
R_{A}=\frac{R_{G} R_{G S}}{R_{G}+R_{G S}}=\frac{2 \times 10^{3} \times 224.43}{2 \times 10^{3}+224.43}=201.79 \Omega, \\
X_{A}=X_{G S}=-8.211 \Omega \\
q_{A}=\frac{R_{A}}{X_{A}}=\frac{201.79}{8.211}=-24.576 \Omega .
\end{gathered}
$$

The input series resistance $r_{A}$ and reactance $x_{A}$ for section $A$ are

$$
\begin{gathered}
r_{A}=\frac{R_{A}}{1+q_{A}^{2}}=\frac{201.79}{1+(-24.576)^{2}}=0.33355 \Omega, \\
x_{A}=q_{A} r_{A}=(-24.576) \times 0.33355=-8.1973 \Omega .
\end{gathered}
$$

The amplitude $V_{A m}$ of the voltage in the node $A$ equals to the amplitude of $V_{G S m}$. Thus, the amplitude $I_{A m}$ of current flowing into the node $A$ for the assumed amplitude $V_{G S m}$ is given by

$$
\begin{gathered}
I_{A m}=\frac{V_{A m}}{\sqrt{r_{A}^{2}+x_{A}^{2}}}=\frac{V_{G S m}}{\sqrt{r_{A}^{2}+x_{A}^{2}}} \\
=\frac{13}{\sqrt{0.33355^{2}+(-8.1973)^{2}}}=1.5846 \mathrm{~A} .
\end{gathered}
$$

Hence, power losses $P_{A}$ and $P_{L f}$ are

$$
\begin{gathered}
P_{A}=\frac{I_{A m}^{2}}{2} r_{A}=\frac{1.5846^{2}}{2} \times 0.33355=0.41877 \mathrm{~W} \\
P_{L f}=\frac{I_{A m}^{2}}{2} r_{f}=\frac{1.5846^{2}}{2} \times 0.085=0.10672 \mathrm{~W}
\end{gathered}
$$

From (18) power $P_{D 1}$ is

$$
P_{D 1}=P_{L f}+P_{A}=0.41877+0.10672=0.52549 \mathrm{~W} .
$$

Resistance $R_{D 1}$ from (17), (18) is equal to

$$
R_{D 1}=\frac{V_{O}^{2}}{P_{D 1}}=\frac{P_{O} R_{L}}{P_{D 1}}=\frac{150 \times 50}{0.52549}=14272.39 \Omega .
$$

Resistance $R_{E}$ is found from (15) as

$$
R_{E}=\frac{R_{L} R_{D 1}}{R_{L}+R_{D 1}}=\frac{50 \times 14272.39}{50+14272.39}=49.825 \Omega .
$$

The input parallel resistance $R_{E}$ and the input series resistance $r_{E}$ for section $E$ are related by the equation

$$
r_{E}=\frac{R_{E}}{1+q_{E}^{2}},
$$

where reactance factor $q_{E}$ is

$$
q_{E}=\frac{R_{E}}{X_{E}}=\frac{x_{E}}{r_{E}} .
$$

From (13) and (33) the capacitive reactance factor $q_{E}$

$$
q_{E}=-\sqrt{\frac{R_{E}}{r_{E}}-1}=-\sqrt{\frac{49.825}{7.8423}}=-2.3137 .
$$

Hence, the input series reactance $x_{E}$ to the right of section $E$ is equal to

$$
x_{E}=q_{E} r_{E}=-2.3137 \times 7.8423=-18.145 \Omega .
$$

The series reactance of section FE is

$x_{F E}=x_{F}-x_{E}=-31.020-(-18.145)=-12.875 \Omega$.

The value of capacitor $C_{S R}$ equals

$$
\begin{gathered}
C_{S R}=\frac{1}{2 \pi f\left|x_{F E}\right|} \\
=\frac{1}{2 \pi \times 6.78 \times 10^{6} \times 12.875}=1.823 \mathrm{nF} .
\end{gathered}
$$

From (35) the input parallel reactance $X_{E}$ to the right of section $E$ is

$$
X_{E}=\frac{R_{E}}{q_{E}}=\frac{49.825}{-2.3137}=-21.535 \Omega .
$$

The input parallel reactance $X_{E}$ is equal to a parallel connection of reactance $X_{C O}$ and the input parallel reactance $X_{D}$ to the right of section $D$

$$
\frac{1}{X_{E}}=\frac{1}{X_{C O}}+\frac{1}{X_{D}}
$$

By substituting (2) to (39) reactance $X_{C o}$ and capacitance $C_{O}$ are found

$$
\begin{gathered}
X_{C O}=\frac{X_{E}}{k}=\frac{-21.535}{0.95}=-22.668 \Omega, \\
C_{O}=\frac{1}{2 \pi f\left|X_{C O}\right|} \\
=\frac{1}{2 \pi \times 6.78 \times 10^{6} \times 22.668}=1.0356 \mathrm{nF} .
\end{gathered}
$$

Reactance $X_{D}$ is from (40)

$$
\begin{gathered}
X_{D}=\frac{X_{E} \cdot X_{C o}}{X_{C O}-X_{E}} \\
=\frac{(-21.535) \times(-22.668)}{(-22.668)-(-21.535)}=-430.85 \Omega .
\end{gathered}
$$

Since $R_{D}=R_{D 1}$ thus, the reactance factor $q_{D}$ of section $D$ equals

$$
q_{D}=\frac{R_{D}}{X_{D}}=\frac{R_{D 1}}{X_{D}}=\frac{14272.39}{-430.85}=-33.126 .
$$




\section{A self-oscillating h.f. power generator with a Class $E$ resonant amplifier}

The input series resistance $r_{D}$ and input series reactance $x_{D}$ to the right of section $D$ are

$$
\begin{gathered}
r_{D}=\frac{R_{D}}{1+q_{D}^{2}}=\frac{R_{D 1}}{1+q_{D}^{2}}=\frac{14272.39}{1+(-33.126)^{2}}=12.995 \Omega \\
x_{D}=q_{D} r_{D}=(-33.126) \times 12.995=-430.47 \Omega .
\end{gathered}
$$

To find $C_{1}, C_{2}$ and $L_{f}$ it is necessary to calculate input series resistances $r_{B}, r_{C}$ and the reactance factors $q_{B}, q_{C}$ for sections $B$ and $C$

$$
\begin{gathered}
r_{C}=r_{D}, \\
r_{B}=r_{A B}+r_{A}=r_{L f}+r_{A} \\
=0.085+0.33355=0.41855 \Omega .
\end{gathered}
$$

As it has been shown in [11] the reactance factors $q_{B}$ and $q_{C}$ can be found from (47) and the following relations:

$$
\begin{gathered}
R_{C}=R_{B}, \\
-\phi_{A G}=-\phi_{E G}-\phi_{C D}-\phi_{A B}, \\
0=\phi_{A G}-\left(\arctan q_{E}-\arctan q_{G}\right)
\end{gathered}
$$

$-\left(\arctan q_{C}-\arctan q_{D}\right)-\left(\arctan q_{A}-\arctan q_{B}\right)$,

where $-\phi_{A G}=3.4209 \mathrm{rad}-$ is equal to the phase shift between the fundamental components of the waveforms $v_{G S}(t)$ and $v_{D S}(t)$ for the nominal operation of the Class $\mathrm{E}$ amplifier $[11,12] ; \phi_{E G}, \phi_{C D}, \phi_{A B}-$ are the phase shifts for the fundamental component between the input and output voltages for the respective sections, $E G, C D$ and $A B$.

The equation (50) is the Barkhausen phase shift criterion for the circuit of Fig. 1a. By assuming

$$
\begin{gathered}
\theta=\arctan q_{C}-\arctan q_{B} \\
=\phi_{A G}-\arctan q_{A}+\arctan q_{D}-\arctan q_{E} \\
+\arctan q_{G}=-3.4209-\arctan (-24.576) \\
+\arctan (-33.126)-\arctan (-2.3137) \\
+\arctan (1.1525)=-1.4124 \mathrm{rad}
\end{gathered}
$$

then from (47), (48), (49) and (50) the reactance factors $q_{B}$, $q_{C}$ are obtained as [11]

$$
\begin{gathered}
q_{C}-\frac{1}{\tan \theta}\left(\sqrt{\frac{r_{B}}{r_{C}}\left(1+\tan ^{2} \theta\right)-1}\right) \\
=\frac{1}{\tan (-1.4124)}\left(\sqrt{\frac{0.41855}{12.995} \times\left(1+\tan ^{2}(-1.4124)\right)-1}\right) \\
=-0.02222 \\
q_{B}=\sqrt{\frac{r_{C}}{r_{B}}\left(1+q_{C}^{2}\right)-1} \\
=\sqrt{\frac{12.995}{0.41855} \times\left(1+(-0.02222)^{2}\right)-1}=5.4830
\end{gathered}
$$

For section $B$ the series input reactance $x_{B}$ and reactance $x_{A B}$ are given as

$$
\begin{gathered}
x_{B}=q_{B} r_{B}=5.4830 \times 0.41855=2.2949 \Omega, \\
x_{A B}=X_{L f}=x_{B}-x_{A} \\
=2.2949-(-8.1973)=10.4922 \Omega .
\end{gathered}
$$

Hence, the feedback inductance $L_{f}$ equals

$$
L_{f}=\frac{X_{L f}}{2 \pi f}=\frac{10.4922}{2 \pi \times 6.78 \times 10^{6}}=246.3 \mathrm{nH} .
$$

The series input reactance $x_{C}$ to the right of section $C$ is

$$
x_{C}=q_{C} r_{C}=-0.02222 \times 12.995=-0.28875 \Omega .
$$

The reactance $X_{C 1}$ of capacitor $C_{1}$ is expressed as

$$
\begin{gathered}
X_{C 1}=x_{C D}=x_{D}-x_{C} \\
=-430.47-(-0.28875)=-430.18 \Omega .
\end{gathered}
$$

The value of capacitor $C_{1}$ is equal to

$$
\begin{gathered}
C_{1}=\frac{1}{2 \pi f\left|X_{C 1}\right|} \\
=\frac{1}{2 \pi \times 6.78 \times 10^{6} \times 430.18}=54.57 \mathrm{pF} .
\end{gathered}
$$

The input parallel resistance $R_{B}$ and reactance $X_{B}$ to the right of section $B$ are

$$
\begin{gathered}
R_{B}=r_{B}\left(1+q_{B}^{2}\right) \\
=0.41855 \times\left(1+5.4830^{2}\right)=13.002 \Omega, \\
X_{B}=\frac{R_{B}}{q_{B}}=\frac{13.002}{5.4830}=2.3713 .
\end{gathered}
$$

Because $R_{C}=R_{B}$ the input parallel reactance $X_{C}$ to the right of section $C$ is

$$
X_{C}=\frac{R_{C}}{q_{C}}=\frac{13.002}{-0.02222}=-585.15 \Omega .
$$

The reactance $X_{C}$ results from a parallel connection of reactance $X_{B}$ and reactance $X_{C 2}$

$$
\frac{1}{X_{C}}=\frac{1}{X_{B}}+\frac{1}{X_{C 2}} .
$$

Thus, reactance $X_{C 2}$ and capacitance $C_{2}$ are found as

$$
\begin{gathered}
X_{C 2}=\frac{X_{B} X_{C}}{X_{B}-X_{C}}=\frac{2.3713 \times(-585.15)}{2.3713-(-585.15)}=-2.3617 \Omega, \\
C_{2}=\frac{1}{2 \pi f\left|X_{C 2}\right|} \\
=\frac{1}{2 \pi \times 6.78 \times 10^{6} \times 2.3617}=9.940 \mathrm{nF} .
\end{gathered}
$$

Power losses in the generator components can be found from the following equations $[11,12]$ :

- de supply current $I_{D D}$

$$
I_{D D}=\frac{P_{S}}{V_{D D}}=\frac{164.835}{48}=3.434 \mathrm{~A},
$$

- amplitude $I_{m}$ of current in $C_{S R}$ and $L_{S R}$

$$
\begin{gathered}
I_{m}=\frac{\sqrt{\pi^{2}+4}}{2} I_{D D} \\
=\frac{\sqrt{\pi^{2}+4}}{2} \times 3.434=6.394 \mathrm{~A},
\end{gathered}
$$

- de power loss in choke $L_{C H}$

$$
P_{L C H}=I_{D D}^{2} r_{L C H}=3.434^{2} \times 0.062=0.7311 \mathrm{~W},
$$


M. Mikołajewski

- conduction losses in the transistor $T$

$$
\begin{gathered}
P_{\text {Tcond }}=\frac{\pi^{2}+28}{16} r_{D S} I_{D D}^{2} \\
=\frac{\pi^{2}+28}{16} \times 0.4 \times 3.434^{2}=11.16 \mathrm{~W},
\end{gathered}
$$

- switching losses in the transistor $T$ for the assumed fall time $t_{\text {fall }}=5 \mathrm{~ns}$ of the drain current $i_{D S}(t)$

$$
\begin{gathered}
P_{\text {Tswitch }}=\frac{\left(2 \pi f t_{\text {fall }}\right)^{2}}{12} P_{S} \\
=\frac{\left(2 \pi \times 6.78 \times 10^{6} \times 5 \times 10^{-9}\right)^{2}}{12} \\
\times 164.835=0.6232 \mathrm{~W},
\end{gathered}
$$

- losses $P_{G S}$ in the gate of the transistor $T$ result from series gate resistance $r_{G S}$ and the gate current with the amplitude $I_{G S m}$

$$
\begin{aligned}
& P_{G S}=\frac{I_{G S m}^{2}}{2} r_{G S}=\frac{V_{G S m}^{2}}{2\left(r_{G S}^{2}+x_{G S}^{2}\right)} r_{G S} \\
& =\frac{13^{2}}{2 \times\left(0.3^{2}+8.2^{2}\right)} \times 0.3=0.3765 \mathrm{~W},
\end{aligned}
$$

- total power losses $P_{\text {Ttot }}$ in the transistor $T$ are

$$
\begin{gathered}
P_{\text {Ttot }}=P_{\text {Tcond }}+P_{\text {Tswitch }}+P_{G S} \\
=11.16+0.6232+0.3765=12.16 \mathrm{~W},
\end{gathered}
$$

- power losses in inductance $L_{S R}$ is

$$
P_{L S R}=\frac{I_{m}^{2}}{2} r_{L S R}=\frac{6.394^{2}}{2} \times 0.22=4.497 \mathrm{~W} .
$$

Parasitic loss resistances of capacitors are usually low if compared to loss resistances in inductances and the switch. Therefore, the influence of the capacitor losses on waveforms and the network impedance in the generator can be neglected thus simplifying the design procedure. However, even low capacitor losses can significantly raise the temperature of a small-size capacitor degrading its parameters. Therefore, it is necessary to estimate these losses as well. The loss resistances of the used in the experimental circuit SMD mica capacitors were estimated from the manufacturer's catalogue (Cornell Dubilier) as follows $r_{C R}=0.02 \Omega, r_{C S R}=0.005 \Omega$, $r_{C O}=0.01 \Omega, r_{C 1}=0.1 \Omega, r_{C 2}=0.001 \Omega$.

Thus, power losses in capacitors $C_{R}, C_{S R}, C_{O}, C_{1}, C_{2}$ are

$$
\begin{gathered}
P_{C R}=\frac{\pi^{2}-4}{16} I_{D D}^{2} r_{C R} \\
=\frac{\pi^{2}-4}{16} \times 3.434^{2} \times 0.02=0.0865 \mathrm{~W}, \\
P_{C S R}=\frac{I_{m}^{2}}{2} r_{C S R}=\frac{6.394^{2}}{2} \times 0.005=0.1022 \mathrm{~W}, \\
P_{C O}=\left(\frac{V_{O}}{\left|X_{C O}\right|}\right)^{2} r_{C O} \\
=\frac{P_{O} R_{L}}{\left|X_{C O}\right|^{2}} r_{C O}=\frac{150 \times 50}{22.668} \times 0.01=0.1460 \mathrm{~W},
\end{gathered}
$$

$$
\begin{gathered}
P_{C 1}=\frac{V_{O}^{2}}{\left(X_{C 1}+x_{C}\right)^{2}+r_{C}^{2}} r_{C 1} \\
=\frac{P_{O} R_{L}}{\left(X_{C 1}+x_{C}\right)^{2}+r_{C}^{2}} r_{C 1} \\
=\frac{150 \times 50}{(-430.18-0.28875)^{2}+12.995^{2}} \\
P_{C 2}=\left(\begin{array}{c}
\left.\frac{\times 0.1=0.0040 \mathrm{~W}}{\sqrt{\left(X_{C 1}+x_{C}\right)^{2}+r_{C}^{2}}} \frac{\sqrt{x_{C}^{2}+r_{C}^{2}}}{X_{C 2}}\right)^{2} r_{C 2} \\
=\frac{V_{O}^{2}}{\left(X_{C 1}+x_{C}\right)^{2}+r_{C}^{2}} \frac{x_{C}^{2}+r_{C}^{2}}{X_{C 2}^{2}} r_{C 2} \\
=\frac{P_{O} R_{L}}{\left(X_{C 1}+x_{C}\right)^{2}+r_{C}^{2}} \frac{x_{C}^{2}+r_{C}^{2}}{X_{C 2}^{2}} r_{C 2} \\
=\frac{150 \times 50}{(-430.18-0.28875)^{2}+12.995^{2}} \\
\frac{(-0.28875)^{2}+12.995^{2}}{(-2.3617)^{2}} \\
\times 0.001=0.0012 \mathrm{~W} .
\end{array}\right.
\end{gathered}
$$

The estimated total power losses in the generator components equals

$$
\begin{gathered}
P_{l}=P_{D 1}+P_{L C H}+P_{\text {Tcond }}+P_{\text {Tswitch }}+P_{G S} \\
+P_{L S R}+P_{C R}+P_{C S R}+P_{C O}+P_{C 1}+P_{C 2} \\
=0.52549+0.7111+11.16+0.6232+0.3765 \\
+4.497+0.0865+0.1022+0.1460 \\
+0.0040+0.0012=18.233 \mathrm{~W} .
\end{gathered}
$$

The generator efficiency $\eta$ obtained from (3) and (79) is

$$
\eta=\frac{P_{S}-P_{l}}{P_{S}}=\frac{164.835-18.233}{164.835} \approx 0.89 .
$$

The calculated efficiency (80) differs only about $2 \%$ from its assumed value.

For the given assumptions the component values were also calculated for the classic Class $\mathrm{E}$ generator $(k=0)$. The obtained results for the classic Class E generator and its modified version of Fig. 1a are presented in Table 1 and compared with the component values used in the experimental generator. The results prove that by the application of capacitor $C_{O}$ it was possible to reduce by 20 times the values of $C_{1}$ and $C_{2}$ with slightly increased value of inductance $L_{f}$. Comparing to the classic circuit the modification does not change the operating frequency of the generator nor its output power. The current and voltage waveforms of the transistor $T$ also remain unchanged. The differences between calculated and experimental values of the components are caused by the assumed low value of $Q_{S R}$ [20] and by difficulties in the precise estimation of nonlinear capacitances of the transistor $T$ and diode $D_{Z}$. Figure 2 and Fig. 3a show the influence of the parameter $k$ on values of $C_{1}, C_{2}, C_{O}$ and $L_{f}$ as well as on normalized current amplitudes in $C_{1}, C_{2}, C_{O}$. By changing $k$ it is possible to optimize the generator with the respect to the 


\section{A self-oscillating h.f. power generator with a Class E resonant amplifier}

values of capacitors $C_{1}, C_{2}, C_{O}$ or the amplitude of current in them.

The proposed circuit of Fig. 1a was built according to the assumptions using inductances made with toroidal carbonyl cores $L_{C H}$ (T106-2), $L_{S R}$ (T200-6), $L_{f}$ (T50-6). Measured total efficiency of the generator was $\eta=0.91$ and resulted mainly from conduction power losses in on-resistance $r_{D S}$ of the transistor switch $T$ as well as losses in inductance $L_{S R}$. Voltage waveforms measured in the circuit (Fig. 3b) agreed well with the assumed $\left(V_{G S m}=13 \mathrm{~V}\right)$ and calculated values $\left(V_{D \text { Speak }}=3.61 V_{D D}=173.3 \mathrm{~V}[20]\right.$, $\left.V_{\text {ORMS }}=\left(P_{O} R_{L}\right)^{1 / 2}=86.6 \mathrm{~V}\right)$.

Table 1

Component values for the Class E generator

\begin{tabular}{cccc}
\hline \hline \multirow{2}{*}{ Component } & \multirow{2}{*}{$\begin{array}{c}\text { classic generator } \\
\text { calculated values }\end{array}$} & \multicolumn{2}{c}{$\begin{array}{c}\text { values for the proposed } \\
\text { circuit of Fig. 1a }\end{array}$} \\
\cline { 3 - 4 } & $534.57 \mathrm{pF}$ & $534.57 \mathrm{pF}$ & $390 \mathrm{pF}$ \\
\hline$C_{R}$ & $946.3 \mathrm{nH}$ & $946.3 \mathrm{nH}$ & $944.8 \mathrm{nH}$ \\
\hline$L_{S R}$ & $1.823 \mathrm{nF}$ & $1.823 \mathrm{nF}$ & $1 \mathrm{nF}+820 \mathrm{pF}$ \\
\hline$C_{S R}$ & $50 \Omega$ & $50 \Omega$ & $50 \Omega$ \\
\hline$R_{L}$ & - & $1.0356 \mathrm{nF}$ & $1 \mathrm{nF}$ \\
\hline$C_{O}$ & $1.095 \mathrm{nF}$ & $54.57 \mathrm{pF}$ & $51 \mathrm{pF}$ \\
\hline$C_{1}$ & $199.6 \mathrm{nF}$ & $9.940 \mathrm{nF}$ & $4 * 2.2 \mathrm{nF}+1 \mathrm{nF}+3 * 390 \mathrm{pF}$ \\
\hline$C_{2}$ & $193.9 \mathrm{nH}$ & $246.3 \mathrm{nH}$ & $226 \mathrm{nH}$ \\
\hline$L_{f}$ & & &
\end{tabular}

a)

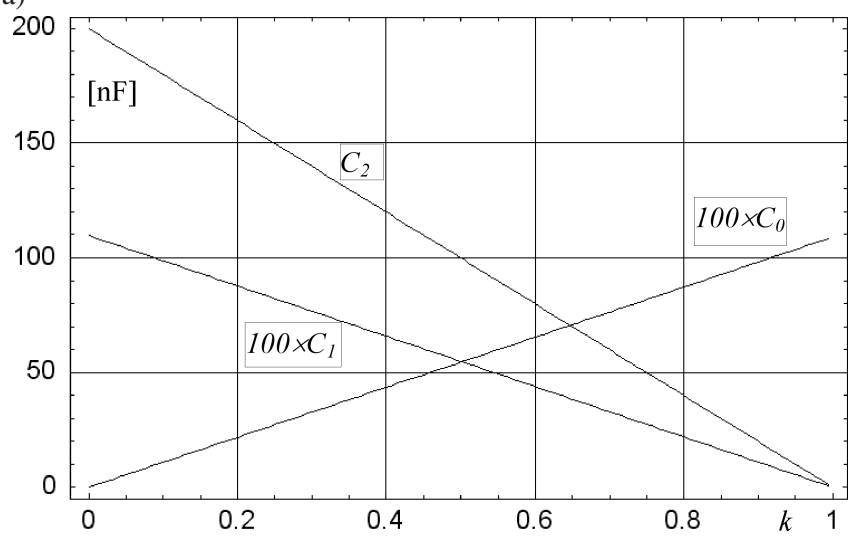

b)

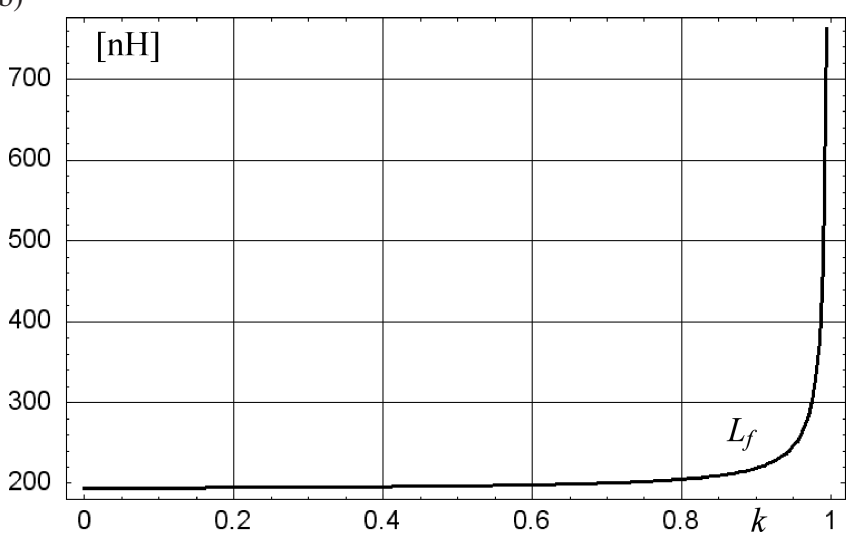

Fig. 2. Calculated values of capacitors $C_{1}, C_{2}, C_{O}$ (a) and inductance $L_{f}$ (b) versus $k$ in the designed generator a)

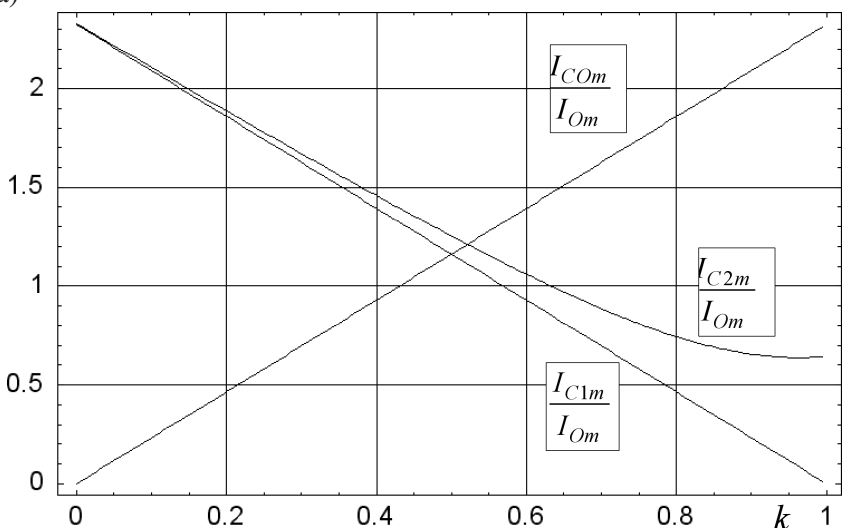

b)

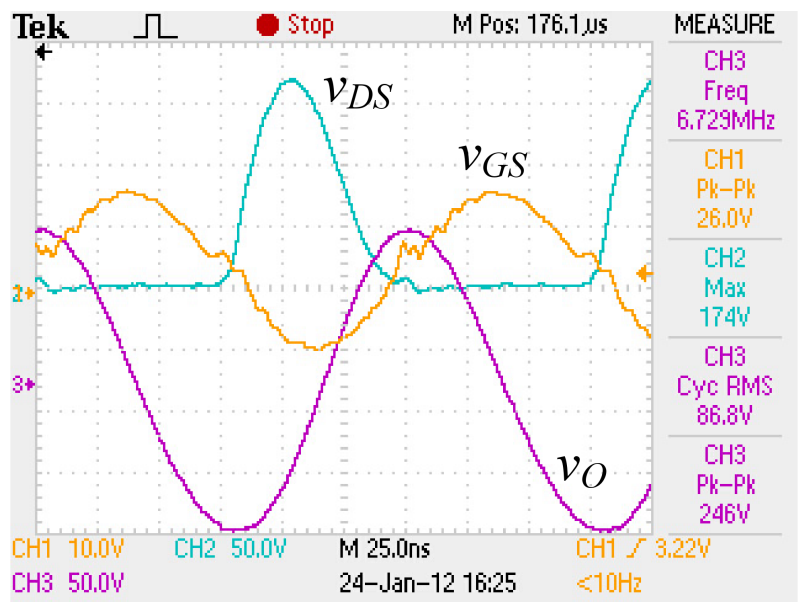

Fig. 3. Calculated normalized amplitudes of current in capacitors $C_{1}, C_{2}, C_{O}$ versus $k$ in the designed generator (a) and measured voltage waveforms in the experimental circuit $\left(I_{O m}-\right.$ amplitude of current in load resistance $R_{L}$ )

The significant decrease of the capacitor $C_{2}$ value in the experimental generator also reduces the time constant $\tau_{G} \approx R_{G}\left(C_{i s s}+C_{2}\right)$ of the voltage $V_{G S 0}$ rise in the gate circuit $\left(C_{i s s}\right.$ - the input capacitance of the transistor switch). Therefore it simplifies the application of the burst control method in the generator (Fig. 4). The burst control of output power is achieved by means of transistor switch $S$. The voltage $v_{C O N T R}$ high state turns on switch $S$, which short circuits transistor $T$ gate and stops oscillations in the generator. When voltage $v_{C O N T R}$ is low the switch $S$ turns off and the voltage $V_{G S 0}$ rises with the time constant $\tau_{G}$ till it achieves the threshold value $V_{G S t h}$. Then oscillations are initiated. By regulating the ratio of the switch $S$ turn-on time to its turnoff time the generator output power can be controlled in the whole range with high efficiency. Diodes $D_{Z 1}, D_{1}$ protect the transistor $T$ from breakdown by the high peak value of $v_{D S}$ voltage which appears when the transistor $T$ is turned off by the switch $S$. The high value of $v_{D S}$ results from the resonant discharge of energy accumulated in supply choke $L_{C H}$ during the time interval when the generator produces h.f. output power $[16,17]$. 
M. Mikołajewski

a)

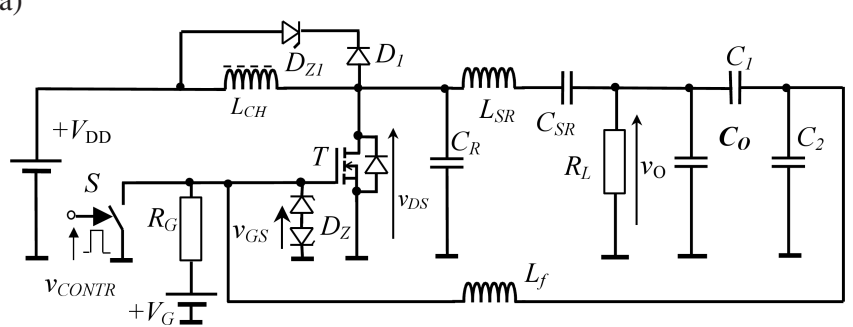

b)

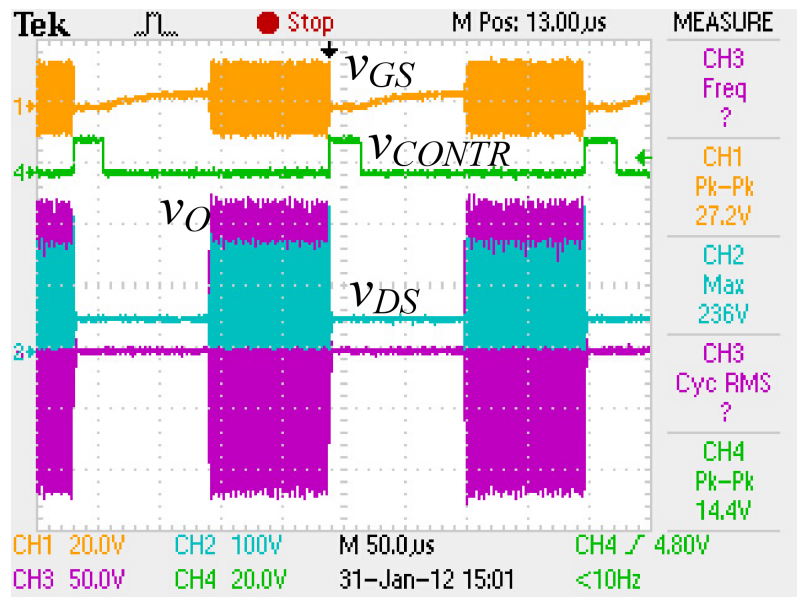

Fig. 4. Burst control of output power in the self-oscillating Class E generator. Circuit diagram (a) and measured voltage waveforms (b)

\section{Conclusions}

The described modification of the Class E generator increases the degree of freedom in designing the circuit. By decreasing the required values of capacitors in the feedback network a reduction of cost in the generator is possible. Moreover, the presented output power control by the burst control method ensures output power regulation in the generator in the whole range with high efficiency. The generator can find applications in high-efficiency h.f. industrial power generators.

\section{REFERENCES}

[1] H. Sarnago, A. Mediano, D. Palacios, and A. Santolaria, "A novel class E RF self-oscillating topology for induction heating applications", Proc. $14^{\text {th }}$ Eur. Conf. on Power Electronics and Appl. (EPE 2011) 1, CD-ROM (2011).

[2] D. Petreus, A. Grama, S. Cadar, E. Plaian, and A. Rusu, "Design of a plasma generator based on E power amplifier and impedance matching", Proc. $12^{\text {th }}$ Int. Conf. on Optimization of Electrical and Electronic Equip., OPTIM 2010 1, 13171322 (2010).

[3] V.G. Krizhanowski, D.V. Chernov, and M.K. Kazimierczuk, "Low-voltage electronic ballast based on Class E oscillator", IEEE Trans. on Power Electronics 22 (3), 863-870 (2007).
[4] C. Thongsongyod, A. Nathakaranakule, and I. Boonyaroonate, "Design of Class E inverter electronic ballast for $35 \mathrm{~W}$ automotive HID lamp", Proc. ECTI-CON 2008 1, 1041-1044 (2008).

[5] A. Georgiadis and A. Collado, "Improving range of passive RFID tags utilizing energy harvesting and high efficiency Class-E oscillators", Proc. $6^{\text {th }}$ Eur. Conf. on Antennas and Propagation (EUCAP) 1, 3455-3458 (2011).

[6] P. Dziurdzia, M. Mysiura, and A. Gołda, "Low voltage integrated converter for waste heat thermoelectric harvesters", Metrol. Meas. Syst. XIX (1), 159-168 (2012).

[7] R.M. Miskiewicz and A.J. Moradewicz, "Contactless power interface for plug-in electric vehicles in V2G systems", Bull. Pol. Ac.: Tech. 59 (4), 561-568 (2011).

[8] H. Hase, H. Sekiya, J. Lu, and T. Yahagi, "Resonant DC-DC converter with Class-E oscillator", IEEE Trans. Circ. Syst. - I 53 (9), 2023-2025 (2006).

[9] T.M. Andersen, S.K. Christensen, A. Knott, and M.A.E. Andersen, "A VHF Class E DC-DC converter with self-oscillating gate driver", Proc. 26th APEC 1, 885-891 (2011).

[10] R.M. Moradewicz and M.P. Kaźmierkowski, "Contacless energy transfer system with FPGA - controlled resonant converter", Bull. Pol. Ac.: Tech. 57 (9), 3181-3190 (2010).

[11] M.K. Kazimierczuk, V.G. Krizhanovski, J.V. Rassokhina, and D.V. Chernov, "Class-E MOSFET tuned power oscillator design procedure", IEEE Trans. Circ. Syst. 52 (6), 1138-1147 (2005).

[12] M.K. Kazimierczuk and D. Czarkowski, Resonant Power Converters, Wiley, New York, 1995.

[13] M.K. Kazimierczuk, V.G. Krizhanovski, J.V. Rassokhina, and D.V. Chernov, "Injection-locked Class-E oscillator", IEEE Trans. Circ. Syst. I 53 (6) 1214-1222 (2006).

[14] J. Ebert and M.K. Kazimierczuk, "Class-E high-efficiency tuned power oscillator", IEEE J. Solid-State Circuits 16 (2), 62-66 (1981).

[15] H.-N. Toussaint, "Transistor power oscillator electronically tunable from 250 to $500 \mathrm{MHz}$ ", Proc. IEEE 56 (2), 226-227 (1968).

[16] M. Mikołajewski, "A Class E amplifier for induction heating with burst control of output power", Proc. PES-7, 49-52 (2009), (in Polish).

[17] M. Mikołajewski, "A Class E amplifier with output power burst control", Electrotechnical News 11, 50-52 (2011), (in Polish).

[18] M.K. Kazimierczuk, "A new aproach to the design of tuned power oscillators", IEEE Trans. Circ. Syst. CAS-29 (4), 261267 (1982)

[19] M.K. Kazimierczuk and D. Murthy-Bellur, "Synthesis of LC oscillators", Int. J. Electrical Engineering Education 49 (1), 26-31 (2012).

[20] M.K. Kazimierczuk and K. Puczko, "Exact analysis of class E tuned power amplifierat any Q and switch duty cycle", IEEE Trans. Circ. Syst. 34 (2), 149-159 (1987) 